\title{
Cancer-associated fibroblasts do not respond to combined irradiation and kinase inhibitor treatment
}

\author{
ANNETTE AFFOLTER $^{1}$, IRENE SCHMIDTMANN ${ }^{2}$, WOLF J. MANN ${ }^{1}$ and JÜRGEN BRIEGER ${ }^{1}$ \\ ${ }^{1}$ Department of Otorhinolaryngology, Head and Neck Surgery, ${ }^{2}$ Institute of Medical Biostatistics, Epidemiology \\ and Informatics, University Medical Center of the Johannes Gutenberg University, D-55101 Mainz, Germany
}

Received October 3, 2012; Accepted November 15, 2012

DOI: $10.3892 /$ or.2012.2180

\begin{abstract}
The emergence of radioresistance is a significant issue in the treatment of squamous cell carcinoma. We recently demonstrated that post-radiogenic extracellular signal-regulated kinase (ERK) signaling might decrease radiosensitivity in this cancer type. To further elucidate how tumor-organizing cell types respond to irradiation and ERK pathway inhibition, we analyzed one oral squamous cell carcinoma and one lung cancer cell line (HNSCCUM-02T, A549), fibroblasts (NIH3T3), primary normal and cancer-associated fibroblasts (CAFs) in vitro. Irradiated cells treated with mitogen-activated protein kinase (MAPK) inhibitor U0126 were screened for $\mathrm{pERK}$ levels. Post-radiogenic cellular responses were functionally analyzed by proliferation and colony assays. We found analogous pERK expression, proliferation and survival of tumor and normal fibroblast cells. CAFs did not show any response to treatment. We hypothesized that radiation and MAPK inhibition have no dose-limiting effect on tumor-surrounding normal tissue. As CAFs are considered to influence the radioresponse of the entire tumor, but are not affected by treatment themselves, potential CAF-mediated tumor protection should be considered in further studies.
\end{abstract}

\section{Introduction}

Aberrant activities of interrelated signaling pathways contribute to squamous cell carcinoma (SCC) development and are the focus of several molecular targeted therapy approaches, such as epidermal growth factor pathway and tyrosine kinase inhibitors. In this regard, particularly the mechanisms of inherent radioresistance in this entity remain to be defined. We recently showed that the MAPK pathway, which can be

Correspondence to: $\mathrm{Dr}$ Annette Affolter, Department of Otorhinolaryngology, Head and Neck Surgery, Molecular Tumor Biology Laboratory, Building 102, University Medical Center, Langenbeckstrasse 1, D-55101 Mainz, Germany

E-mail: annette.affolter@web.de

Key words: radiation resistance, cancer-associated fibroblasts, mitogenactivated protein kinase pathway, oral squamous cell carcinoma, in vitro tumor model upregulated by ionizing radiation (IR), seems to play a pivotal role in irradiation resistance development (1). The cascade is involved in cell growth, thereby conferring a survival advantage $(2,3)$. ERK activation induced by IR is assumed to lead to an increase of vascular endothelial growth factor (VEGF) expression levels and, therefore, to a feedback loop of radioresistance (4).

Our present study focuses on the role of the tumor microenvironment in radioresistance development. A benefit of IR as a therapeutic tool is the locoregional application, which prevents systemic toxicity. However, besides targeting cancer cells, IR affects surrounding normal cells, which is a doselimiting factor. Unknown effects may occur particularly from the response of tumor-infiltrating fibroblasts.

There is increasing knowledge that solid tumors do not only contain neoplastic cells, but are rather assembled of a mixture of cells and extracellular matrix constituting the tumor stroma (5). Cancer-associated fibroblasts (CAFs) are known to promote growth and invasion of cancer cells by various mechanisms (6), maintaining a permanent crosstalk with tumor cells. They are therefore considered a key player in cancer progression and, similarly, a promising target in therapy regimes. In this regard, it has already been shown that exposure to lower doses of IR ( $<20 \mathrm{~Gy}$ ) enhances the capacity of senescent fibroblasts to promote the survival of co-cultured cancer cells (7).

In the present study we continued to explore cellular responses to a combination therapy regimen. Tumor and fibroblast cell lines were used as models for cancer and stroma cells and were treated with the specific MEK/ERK inhibitor U0126 before irradiation in an attempt to mimic the therapeutical situation. CAFs were analyzed in order to depict potential interference mechanisms of this cellular subgroup in response to treatment. Our aim was to investigate the impact of irradiation and kinase inhibition on tumor and normal cells, including CAFs, focusing on their colony forming and proliferative properties. Our data contribute to a better understanding of the responses of CAFs to avoid unwanted responses when developing therapeutic strategies.

\section{Materials and methods}

Tumor cell lines. The oral squamous cell carcinoma (OSCC) cell line HNSCCUM-02T was previously established and 


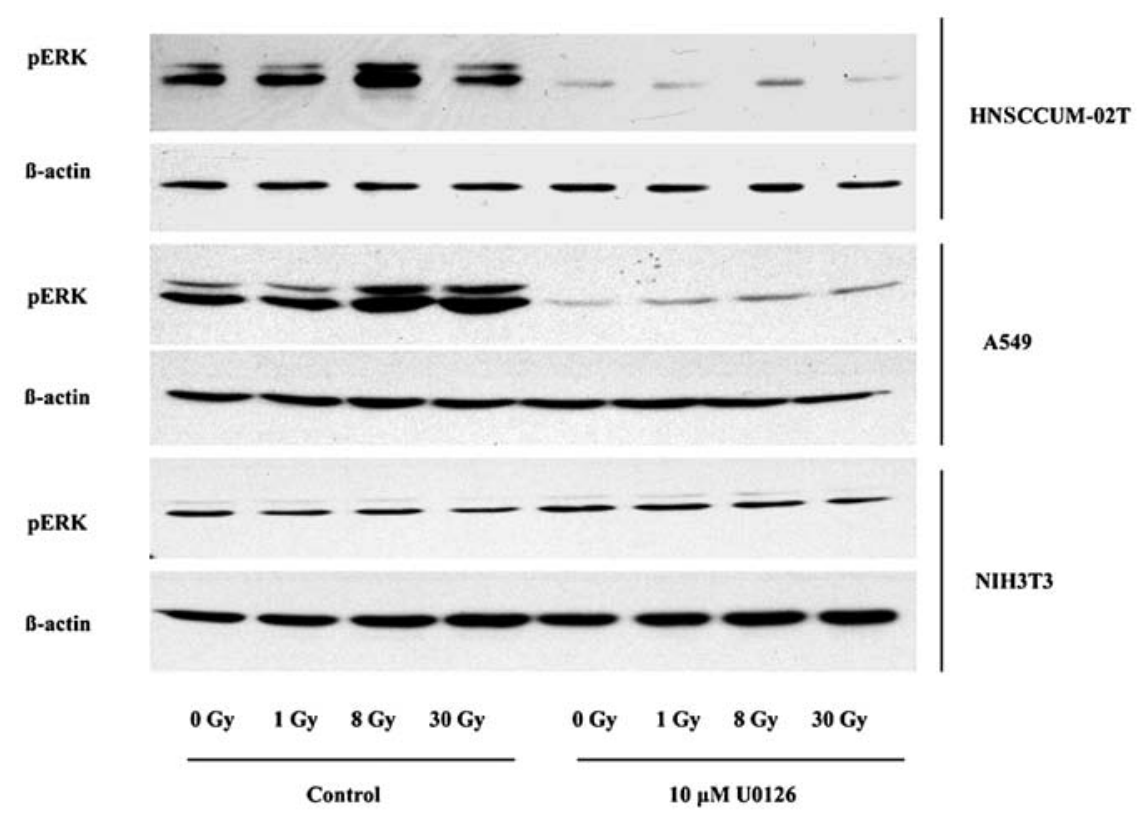

Figure 1. Effect of IR and inhibitor treatment on expression levels of pERK in HNSCCUM-02T, A549 and NIH3T3. Cells were irradiated with intensities of 1, 8 and $30 \mathrm{~Gy}$. Mock-irradiated cells served as control. Western blots of pERK and $\beta$-actin are shown. For inhibitor analysis, U0126 was added. HNSCCUM-02T and A549 showed elevated post-radiogenic pERK expression. pERK was almost completely suppressed by inhibitor treatment in tumor cells. NIH3T3 showed only minor responses to treatment.

characterized in our laboratory (8). Non-small cell lung cancer (NSCLC) cell line A549 and mouse embryonic fibroblast cell line NIH3T3 were purchased from ATCC (Manassas, VA, USA). Fibroblast cell lines derived from OSCC and normal tissues were established and cultured in our laboratory. Experiments were approved by the relevant ethics committee. Cells were maintained in DMEM (Gibco ${ }^{\circledR}$ Invitrogen, Auckland, NZ) supplemented with 5\% FCS (PAA Laboratories, Cölbe, Germany) and antibiotics (penicillin $100 \mathrm{U} / \mathrm{ml}$ and streptomycin $100 \mu \mathrm{g} / \mathrm{ml}$ (Gibco Invitrogen) at $37^{\circ} \mathrm{C}$ in $5 \% \mathrm{CO}_{2}$.

Inhibitor treatment and irradiation of cell cultures. Tumor cell lines were isolated by tryptic digestion (PAA Laboratories), stained with trypan blue (Sigma, Taufkirchen, Germany) and counted. Cells $\left(7 \times 10^{5}\right)$ were seeded per T25 flask and cultured in DMEM with 5\% FCS and antibiotics. The cells were serumstarved in DMEM containing antibiotics for $24 \mathrm{~h}$. U0126 (Cell Signaling Technology Inc., Danvers, MA, USA) was added in a final concentration of $10 \mu \mathrm{M}$. After incubation for $2 \mathrm{~h}$, cells were irradiated with intensities of 1,8 and $30 \mathrm{~Gy}$, using a $\gamma$-source $\left(\mathrm{Cs}^{137}\right)$. After $5 \mathrm{~min}$, cells were harvested. Controls were not irradiated.

Sodium dodecyl sulfate-polyacrylamide gel electrophoresis (SDS-PAGE) and western blotting. A volume of homogenate containing $20 \mu \mathrm{g}$ of total protein was loaded onto $12.5 \%$ acrylamide gels and subjected to SDS-PAGE. Gels were transferred to nitrocellulose and were western blotted using an antiphospho-ERK1/2 antibody (R\&D, Minneapolis, MN, USA) and an anti- $\beta$-actin antibody (Sigma) as a loading control. Blots were developed using Western Lightning Chemiluminescence Reagent Plus (Perkin-Elmer Life Sciences, Boston, MA, USA) and AGFA Curix HT 1.000 plus X-ray film. Densitometric analysis for ECL blots was performed using the Corel
Photo-Paint X3 Image system and the respective software and band densities were normalized to that of $\beta$-actin in the same sample. Blots were digitally scanned using Epson Smart Panel (Epson, Munich, Germany). Each experiment was performed in triplicate.

Clonogenic assay and data analysis. Monolayers ( $80 \%$ confluence) of cell line NIH3T3 were dispersed by tryptic digestion (PAA Laboratories) and re-suspended in $10 \mathrm{ml}$ (240 and 720 cells $/ \mathrm{ml}$ for doses of 6 and $8 \mathrm{~Gy}$, respectively) of standard media supplemented with FCS and antibiotics. U0126 (Cell Signaling Technology Inc.) was added at a final concentration of $10 \mu \mathrm{M}$. Following incubation for $2 \mathrm{~h}$, cells were irradiated with the following doses: $0.5,1,2,4,6$ and $8 \mathrm{~Gy}$, using a $\gamma$-source $\left(\mathrm{Cs}^{137}\right)$. Mock-irradiated cultures were processed in parallel. Cells were seeded into T25 flasks and cultured for 10 days. After fixation with ethanol/acetone $(50 \%, v / v)$, cells were stained with $10 \%$ Giemsa (Sigma, St. Louis, MO, USA). Colony formation was defined as a colony of $>50$ cells. Each experiment was performed in duplicate and repeated three times. Survival curves were then created. We examined the proportion of cells at $0 \mathrm{~Gy}$ that were still present at a given dose. The purpose of the statistical analysis was to model the dependence of the proportion of surviving tumor cells on the type of experiment (DMSO or U0126) and on the radiation dose. We assumed a linear-quadratic relationship between log (proportion) of surviving cells and radiation dose.

We took repeated measurements on assays into account by first fitting a linear mixed model with SAS PROC Mixed. We found, however, that the assay variance components were estimated zero. Therefore, we assumed independence of measurements and fitted a linear quadratic model using SAS PROC GLM. We used SAS PROC GLMSelect to determine what degree of polynomial was suitable for the data and 
whether common constant, linear or quadratic terms might be assumed. The statistical analysis was performed using SAS 9.3 (SAS Institute Inc., Cary, NC, USA).

Alamar blue proliferation assays. In addition to the HNSCCUM-02T, A549 and NIH3T3 cell lines, we also used CAFs and fibroblasts derived from normal tissue for this experiment.

Cell proliferation under the influence of IR and U0126 application was assessed with the oxidation-reduction sensitive dye Alamar Blue (Biozol, Eching, Germany), a fluorometric/colorimetric indicator of metabolic activity. Briefly, cells were seeded into T25 flasks $\left(7 \times 10^{5}\right)$ and incubated at $37^{\circ} \mathrm{C}$ in a $5 \% \mathrm{CO}_{2}$ humidified incubator. After $48 \mathrm{~h}$, cells were treated with $10 \mu \mathrm{M}$ of the U0126 MEK inhibitor and DMSO, respectively, for $2 \mathrm{~h}$. Subsequently, cells were harvested and counted. Cells $(750,000)$ were transferred into falcon tubes in a total volume of $4.5 \mathrm{ml}$ medium and were then irradiated with a dosage of 30 Gy. DMSO-treated cells as control and cells treated with U0126 were processed in parallel.

Following treatment, cells were plated onto a 96-well-plate in triplicate $(25,000$ cells $/ 150 \mu 1)$. Cells treated with $100 \%$ ethanol for $5 \mathrm{~min}$ were used as a death control. Cells were left to attach at $37^{\circ} \mathrm{C}$. After 24 or $72 \mathrm{~h}$, respectively, $10 \%$ Alamar Blue was added and the cells were incubated for $4 \mathrm{~h}$ before recording the fluorescence on a Fluorescence Microplate Reader (Fluoroskan Ascent, Thermo Electron Corp., Dreieich, Germany). Results were given as fluorescence units using a 538-nm excitation filter and a 600-nm emission filter. Each experiment was performed 6 -fold and repeated three times.

\section{Results}

MEK/ERK inhibitor U0126 reduces pERK expression in irradiated tumor cells, but not in fibroblasts. We previously reported that radiation-induced phosphorylation of ERK was distinctly diminished after U0126 application in HNSCCUM-02T cells when performing dose course experiments with different IR intensities and treatment with $10 \mu \mathrm{M}$ U0126 followed by immunoblotting (4). In order to verify whether the other cell lines might express post-radiogenically elevated pERK (activated ERK) levels as well, we irradiated with dosages of $0,1,8$ and $30 \mathrm{~Gy}$. The oral cancer cell line HNSCCUM-02T displayed elevated pERK levels after irradiation compared to mock-irradiated cells with maximum levels when treated with 8 Gy. HNSCCUM-02T cells showed a $117 \%$ increase of pERK after an intensity of $8 \mathrm{~Gy}$ in comparison to mock-irradiated cells. Phospho-ERK levels were markedly suppressed after treatment with U0126 throughout all the applied intensities in HNSCCUM-02T. Cells showed a decrease of $86 \%$ in their pERK expression when applying the inhibitor without irradiation, in comparison with untreated cells, and a decrease of $96 \%$ after utilization of 30 Gy in combination with U0126. Similar to the OSCC cells, the A549 NSCLC cell line displayed maximum levels of activated ERK after an irradiation dosage of $8 \mathrm{~Gy}$. Compared to the mock-irradiated cells, we observed a 55\% increase in pERK levels. A549 displayed an average decrease of $89 \%$ in pERK levels after U0126 treatment without irradiation and a pERK reduction of $89 \%$ when applying a $30-$ Gy intensity. However,
Table I. Western blots (mean values and SEM of densitometric analyses).

A, Effect of IR and U0126 treatment on expression levels of pERK in HNSCCUM-02T

\begin{tabular}{lrr}
\hline HNSCCUM-02T & Mean value & \multicolumn{1}{c}{ SEM } \\
\hline 0 Gy & 194,211 & 44,162 \\
$1 \mathrm{~Gy}$ & 259,657 & 89,303 \\
$8 \mathrm{~Gy}$ & 421,151 & 91,338 \\
$30 \mathrm{~Gy}$ & 327,781 & 166,389 \\
$0 \mathrm{~Gy}+\mathrm{U} 0126$ & 26,846 & 22,973 \\
$1 \mathrm{~Gy}+\mathrm{U} 0126$ & 13,112 & 6,189 \\
$8 \mathrm{~Gy}+\mathrm{U} 0126$ & 20,797 & 7,146 \\
$30 \mathrm{~Gy}+\mathrm{U} 0126$ & 7,533 & 4,294 \\
& & \\
\hline
\end{tabular}

B, Effect of IR and U0126 treatment on expression levels of pERK in A549

\begin{tabular}{lrr}
\hline A549 & Mean value & \multicolumn{1}{c}{ SEM } \\
\hline 0 Gy & 674,832 & 199,646 \\
$1 \mathrm{~Gy}$ & 654,505 & 141,277 \\
$8 \mathrm{~Gy}$ & $1,046.941$ & 178,202 \\
$30 \mathrm{~Gy}$ & $1,027.477$ & 216,754 \\
$0 \mathrm{~Gy}+\mathrm{U} 0126$ & 72,807 & 34,964 \\
$1 \mathrm{~Gy}+\mathrm{U} 0126$ & 83,556 & 20,153 \\
$8 \mathrm{~Gy}+\mathrm{U} 0126$ & 99,122 & 31,535 \\
$30 \mathrm{~Gy}+\mathrm{U} 0126$ & 77,410 & 32,684 \\
\hline
\end{tabular}

C, Effect of IR and U0126 treatment on expression levels of pERK in NIH3T3

\begin{tabular}{lcr}
\hline NIH3T3 & Mean value & SEM \\
\hline 0 Gy & 570,900 & 487,118 \\
$1 \mathrm{~Gy}$ & 841,258 & 907,611 \\
$8 \mathrm{~Gy}$ & 449,025 & 276,554 \\
$30 \mathrm{~Gy}$ & 542,448 & 641,098 \\
$0 \mathrm{~Gy}+\mathrm{U} 0126$ & 359,449 & 163,519 \\
$1 \mathrm{~Gy}+\mathrm{U} 0126$ & 355,130 & 83,697 \\
$8 \mathrm{~Gy}+\mathrm{U} 0126$ & 260,587 & 43,929 \\
$30 \mathrm{~Gy}+\mathrm{U} 0126$ & 200,813 & 44,367 \\
\hline
\end{tabular}

NIH3T3 mouse fibroblasts did not respond to lower dosages of 1 and 8 Gy with or without U0126 application. Only after U0126 application at a maximum radiation dosage of $30 \mathrm{~Gy}$ and U0126 was there a minor decrease of pERK levels of $65 \%$ compared to untreated cells (Fig. 1). Table I shows the mean values and SEM of densitometric measurements.

Impact of irradiation and U0126 application on clonogenic survival rates. Clonogenic assays of NIH3T3 cells were 


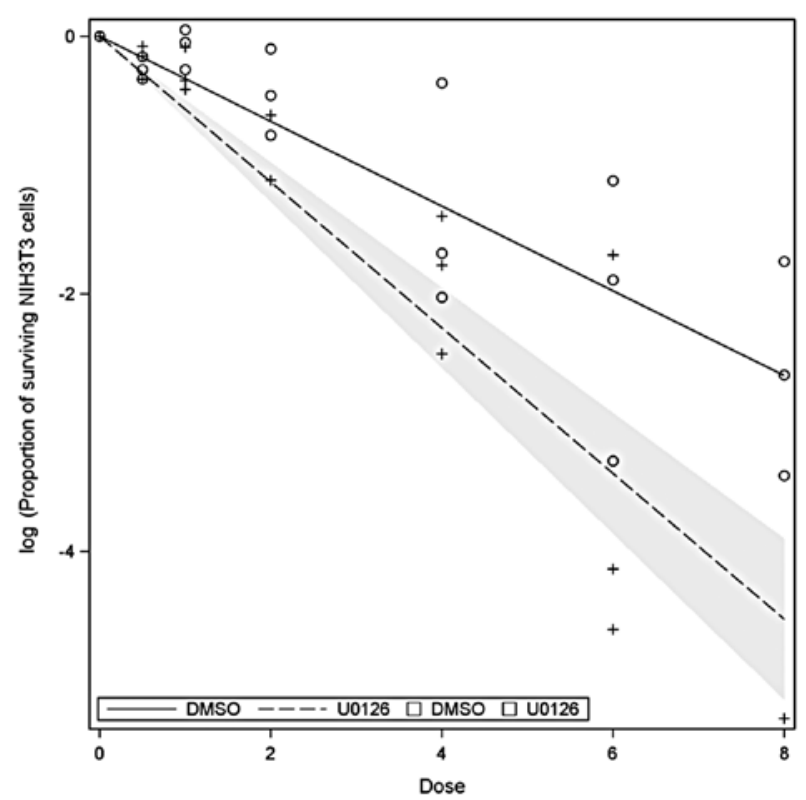

Figure 2. Decrease of clonogenic survival by IR and application of U0126 in NIH3T3. NIH3T3 cells (with and without inhibitor application) treated with increasing dosages of ionizing radiation. There was a statistically significant difference $(\mathrm{p}<0.0001)$. The shaded areas mark the $95 \%$ confidence band for regression line.

performed with intensities ranging from 0.5 to $8 \mathrm{~Gy}$ and compared to non-irradiated cultures. U0126 was added and compared to unstimulated samples. When fitting a linear quadratic model to the $\log$ (proportion) of the surviving cells, the corresponding p-values are all $>0.18$. Using a simpler model involving only linear terms, we found $\mathrm{R}^{2}=0.82$. The coefficients for DMSO and U0126 can clearly be distinguished $(\mathrm{p}<0.0001)$. The resulting functions are displayed in Fig. 2.

Irradiation and U0126 application impede the proliferation of cancer cells and normal fibroblasts but not of CAFs. There was no effect of IR or inhibitor treatment seen after $24 \mathrm{~h}$ of incubation in proliferation (MTT) assays. However, after $72 \mathrm{~h}$, a dosage of $30 \mathrm{~Gy}$ caused a decrease in the disposition of cells to proliferate in both inhibitor-treated and untreated samples. Tumor cell lines HNSCCUM-02T and A549 both showed minor decrease in their proliferative activity when treated with U0126 without IR. When irradiated with $30 \mathrm{~Gy}$, there was a strong decrease of proliferating cells with and without inhibitor application in both tumor cell types compared to the control (HNSCCUM-02T: $30 \mathrm{~Gy}+$ DMSO: 61\% decrease, $30 \mathrm{~Gy}+$ U0126: 56\% decrease; A549: 30 Gy + DMSO: 47\% decrease, $30 \mathrm{~Gy}+\mathrm{U} 0126: 48 \%$ decrease). The proliferation pattern of mouse embryonic fibroblasts, NIH3T3, was similar to those of the cancer cell lines (30 Gy + DMSO: $48 \%$ decrease, $30 \mathrm{~Gy}+$ U0126: 55\% decrease, respectively, compared to the control). However, fibroblasts deriving from non-cancerous human tissue were affected to a lesser extent when treated with $30 \mathrm{~Gy}$ \pm U0126 (30 Gy + DMSO: 26\% decrease, $30 \mathrm{~Gy}+\mathrm{U} 0126$ : $23 \%$ decrease, respectively, compared to the control). However, when looking at the CAFs, we did not detect any notable suppression of proliferative activity either by IR alone or in combination with kinase inhibitor U0126 (30 Gy + DMSO: 5\%
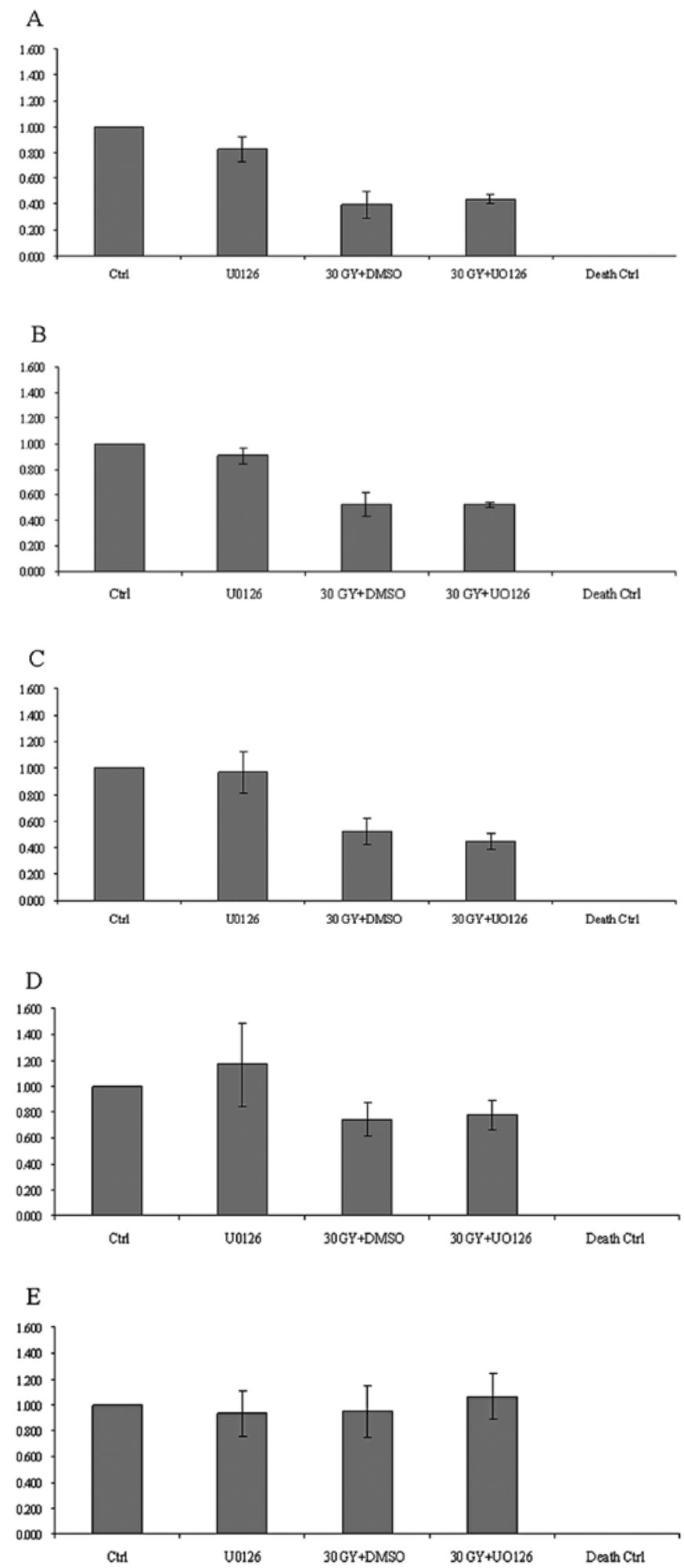

Figure 3. Alamar blue cell viability assays of cancer cells, normal fibroblasts and CAFs. HNSCCUM-02T (A), A549 (B), NIH3T3 (C), fibroblasts derived from normal tissue (D) and CAFs (E) were analyzed. Cells were treated with $10 \mu \mathrm{M}$ of U0126 MEK inhibitor and DMSO, respectively, for $2 \mathrm{~h}$, irradiated with $30 \mathrm{~Gy}$, then incubated for $72 \mathrm{~h}$. Tumor and normal fibroblast cell lines showed analogous reactions to treatment, whereas CAFs did not respond.

decrease, 30 Gy + U0126: 6\% increase, respectively, compared to the control) (Fig. 3). Table II shows the mean values and SEM of densitometric measurements. 
Table II. Proliferation assays (mean values and SEM of densitometric analyses).

A, Effect of IR and U0126 treatment on the proliferative activity of HNSCCUM-02T

\begin{tabular}{lcl}
\hline HNSCCUM-02T & Mean value & SEM \\
\hline Ctrl & 1 & 0 \\
U0126 & 0.824 & 0.1 \\
30 Gy + DMSO & 0.394 & 0.1 \\
30 Gy + UO126 & 0.437 & 0.03 \\
Death ctrl & -0.002 & 0 \\
\hline
\end{tabular}

B, Effect of IR and U0126 treatment on the proliferative activity of A549

\begin{tabular}{lcl}
\hline A549 & Mean value & SEM \\
\hline Ctrl & 1 & 0 \\
U0126 & 0.908 & 0.06 \\
30 Gy + DMSO & 0.523 & 0.1 \\
30 Gy + UO126 & 0.532 & 0.02 \\
Death ctrl & -0.003 & 0 \\
\hline
\end{tabular}

C, Effect of IR and U0126 treatment on the proliferative activity of NIH3T3

\begin{tabular}{lcl}
\hline NIH3T3 & Mean value & SEM \\
\hline Ctrl & 1 & 0 \\
U0126 & 0.969 & 0.16 \\
30 Gy + DMSO & 0.525 & 0.1 \\
30 Gy + UO126 & 0.447 & 0.06 \\
Death ctrl & -0.002 & 0 \\
\hline
\end{tabular}

D, Effect of IR and U0126 treatment on the proliferative activity of normal fibroblasts

\begin{tabular}{lcl}
\hline Normal fibroblasts & Mean value & SEM \\
\hline Ctrl & 1 & 0 \\
U0126 & 1.165 & 0.32 \\
30 Gy + DMSO & 0.745 & 0.13 \\
30 Gy + UO126 & 0.775 & 0.12 \\
Death ctrl & -0.010 & 0.01 \\
\hline
\end{tabular}

E, Effect of IR and U0126 treatment on the proliferative activity of cancer-associated fibroblasts (CAFs)

\begin{tabular}{lcl}
\hline CAFs & Mean value & SEM \\
\hline Ctrl & 1 & 0 \\
U0126 & 0.934 & 0.18 \\
30 Gy + DMSO & 0.951 & 0.2 \\
30 Gy + UO126 & 1.063 & 0.18 \\
Death ctrl & -0.006 & 0.01 \\
\hline
\end{tabular}

\section{Discussion}

Two main obstacles of radiotherapy are acquired radioresistance in cancer cells under therapy and injury of tumor-surrounding tissue. The Ras-MAPK-pathway has proven to be instrumental in the development of radioresistance (9). As this cascade is considered to generate a strong survival signal, specific inhibition of its components is supposed to increase radiosensitivity. Our study provides new evidence on the biological response of tumor-surrounding cells, tumor and stroma cells exposed to irradiation and MAPK inhibitor treatment. We found pERK expression of malignant cells HNSCCUM-02T and A549 almost completely suppressed by U0126 either alone or in combination with IR. In this context, it was interesting to note that NIH3T3 fibroblasts showed marginally diminished pERK levels only after treatment with the maximum dose of $30 \mathrm{~Gy}$ plus inhibitor. Detrimental effects of combination therapy on nearby healthy tissue might not be as substantial as on the tumor mass itself. We recently demonstrated a significantly decreased survival in colony assays for HNSCCUM-02T after application of IR and U0126, whereas A549 showed increased colony counts presumably due to an upstream K-Ras mutation and activation of PI3K/Akt (1).

In the present study, we assayed the colony forming ability of NIH3T3, which displayed analogous responses to HNSCCUM-02T with a relevant reduction of colonies after combined treatment. However, despite the fact that the colony forming ability was suppressed by IR and U0126, the effect was comparable to HNSCCUM-02T, as anticipated from western blotting data. MTT assays revealed similar patterns in HNSCCUM-02T, A549, NIH3T3 and normal fibroblasts. Proliferation was impeded by a dosage of 30 Gy with or without U0126 in these lines. Combined application of IR and MAPK inhibition sufficiently suppressed pERK levels in SCC cells and did not exert any dose-limiting effects on fibroblasts, which is an essential condition for the preservation of healthy surrounding tissue under therapy. However, CAFs did not exhibit any impairment of their proliferation activity, either by treatment with maximum doses of IR or U0126 alone, or after application of both.

In this regard, the responses of tumor-associated fibroblasts to radiation and chemotherapy must be considered. The tumor stroma and particularly CAFs are capable of modulating tumor growth and determine the response to therapy (10). However, only limited data on the potential impact of CAFs on therapy outcome are available. Overall, very few studies have been undertaken using freshly isolated fibroblasts from human cancer specimens $(11,12)$. It is known that breast CAFs stimulated by physiological concentrations of estradiol rapidly induce ERK phosphorylation mediated by estrogen receptor GPR30 (13). If increased expression levels of activated ERK caused by physiological stimuli are existent in oral CAFs as well, this might explain why proliferation of CAFs could not be influenced by MAPK suppression and/or radiation in our system. Beyond that, irradiated CAFs have been reported to release soluble mediators that enhance the invasive potential in pancreatic cancer cells (14). Thus, instead of targeting them, IR seems to lend tumor promoting potential to CAFs. CAFs appear to be intangible to combined therapy approaches successfully 
affecting tumor cells. This might be due to the fact that these cells are conditioned by their tumor environment in a complex mutual interplay, possibly even gaining more resistance towards therapy than tumor cells. Moreover, CAFs are known to harbor a high frequency of genetic alterations that may have already existed independently of the original tumor (15).

We may conclude from our findings that CAFs pose a great challenge for novel strategies targeting cancer. Recent studies even commend using CAFs as therapeutic targets for different carcinoma types (16). Additional studies are needed to better assess the role played by CAFs in initiating and promoting tumorigenic alterations in cancer cells as well as in modifying the response of tumors to therapy. Enhanced understanding of the Gordian network of cancer cell-stroma interactions and the signaling cascades involved in CAF-mediated protection is the prerequisite to modulate treatment resistance of tumor and microenvironment by designing appropriate multitargeting strategies.

\section{Acknowledgements}

The authors thank Ms. Simone Mendler and Ms. Bettina Mros for their excellent technical support. This study was funded by a grant provided by the Foundation Head and Neck Tumor Research, Wiesbaden, Germany.

\section{References}

1. Affolter A, Drigotas M, Fruth K, et al: Increased radioresistance via G12S K-Ras by compensatory upregulation of MAPK and PI3K pathways in epithelial cancer. Head Neck: Feb 2, 2012 (Epub ahead of print) doi: 10.1002/hed.22954.

2. Guyton KZ, Liu Y, Gorospe M, Xu Q and Holbrook NJ: Activation of mitogen-activated protein kinase by $\mathrm{H}_{2} \mathrm{O}_{2}$. Role in cell survival following oxidant injury. J Biol Chem 271: 4138-4142, 1996.

3. Robinson MJ and Cobb MH: Mitogen-activated protein kinase pathways. Curr Opin Cell Biol 9: 180-186, 1997.
4. Affolter A, Fruth K, Brochhausen C, Schmidtmann I, Mann WJ and Brieger $\mathrm{J}$ : Activation of mitogen-activated protein kinase extracellular signal-related kinase in head and neck squamous cell carcinomas after irradiation as part of a rescue mechanism. Head Neck 33: 1448-1457, 2011.

5. Bissell MJ and Radisky D: Putting tumours in context. Nat Rev Cancer 1: 46-54, 2001.

6. Tsai KK, Chuang JJ, Little JB and Yuan ZM: Cellular mechanisms for low-dose ionizing radiation-induced perturbation of the breast tissue microenvironment. Cancer Res 65: 6734-6744, 2005.

7. Tsai KK, Stuart J, Chuang JJ, Little JB and Yuan ZM: Low-dose radiation-induced senescent stromal fibroblasts render nearby breast cancer cells radioresistant. Radiat Res 172: 306-313, 2009.

8. Welkoborsky HJ, Jacob R, Riazimand SH, Bernauer HS and Mann WJ: Molecular biologic characteristics of seven new cell lines of squamous cell carcinomas of the head and neck and comparison to fresh tumor tissue. Oncology 65: 60-71, 2003.

9. Gupta AK, Bakanauskas VJ, Cerniglia GJ, et al: The Ras radiation resistance pathway. Cancer Res 61: 4278-4282, 2001.

10. Chometon $\mathrm{G}$ and Jendrossek V: Targeting the tumour stroma to increase efficacy of chemo- and radiotherapy. Clin Transl Oncol 11: 75-81, 2009.

11. Hwang RF, Moore T, Arumugam T, et al: Cancer-associated stromal fibroblasts promote pancreatic tumor progression. Cancer Res 68: 918-926, 2008.

12. Hawasawi NM, Ghebeh H, Hendrayani SF, et al: Breastcarcinoma-associated fibroblasts and their counterparts display neoplastic-specific changes. Cancer Res 68: 2717-2725, 2008.

13. Madeo A and Maggiolini M: Nuclear alternate estrogen receptor GPR30 mediates $17 \beta$-estradiol-induced gene expression and migration in breast cancer-associated fibroblasts Cancer Res 70: 6036-6046, 2010.

14. Ohuchida K, Mizumoto K, Murakami M, et al: Radiation to stromal fibroblasts increases invasiveness of pancreatic cancer cells through tumor-stromal interactions. Cancer Res 64: 32153222, 2004

15. Littlepage LE, Egeblad M and Werb Z: Coevolution of cancer and stromal cellular responses. Cancer Cell 7: 499-500, 2005.

16. Kalluri R and Zeisberg M: Fibroblasts in cancer. Nat Rev Cancer 6: 392-401, 2006. 